\title{
Coaching cognitivo conductual: una herramienta para los psicólogos
}

\author{
Pedro R. Gil-Monte \\ Unidad de Investigación Psicosocial de la Conducta Organizacional (UNIPSICO) \\ Universitat de València (Valencia, España)
}

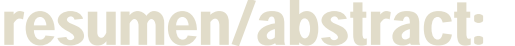

El objetivo de este estudio es presentar una breve revisión de la literatura sobre los principios del coaching cognitivo-conductual, y los principales modelos para su aplicación en la práctica psicológica con el fin de facilitar el ejercicio profesional de los psicólogos en este campo de intervención. El estudio identifica las principales diferencias entre los clientes de coaching (bajos niveles de patología y altos niveles de funcionalidad), y los clientes de psicoterapia (alto nivel de patología y baja funcionalidad). A continuación se presentan las bases teóricas del coaching cognitivo-conductual, que deriva del entrenamiento en resolución de problemas y de la terapia cognitivo-conductual. El siguiente punto ofrece algunas preguntas guía y estrategias que puede utilizar el coach para analizar los pensamientos de los clientes y sus consecuencias. Como modelos de referencia para la práctica profesional el estudio incluye los modelos PRACTICE, SPACE, y ABCDEF. Se concluye con algunas sugerencias para aplicar el coaching para la prevención de riesgos psicosociales en el trabajo.
\end{abstract}

The purpose of this study is to present a brief review of the theoretical basis of cognitive-behavioural coaching. In addition, the study presents the most relevant models to develop the cognitive-behavioural coaching, as a practice tool for psychologist practitioners in this intervention field. The study identifies the main differences between the coaching clients (lower pathology and higher functionality), and psychotherapy clients (higher pathology and lower functionality). Then, the theoretical basis of cognitive behavioral coaching are presented-i.e., problem-solving training, and cognitive behavioral therapy-; and some questions and strategies that the coach would use to analyze the client's irrational thoughts, and their consequences. As reference models for practitioners, the study describes the PRACTICE, SPACE and ABCDEF models. As conclusions, the study offers some suggestions to develop the practice of coaching as a tool in occupational risks prevention.

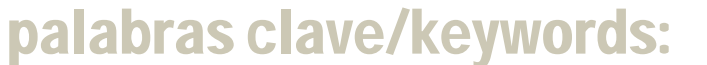

coaching, cognitivo-conductual, salud ocupacional coaching, cognitive-behavioural, occupational health

\section{Coaching cognitivo conductual: una herramienta para los psicólogos}

La cultura del coaching se ha extendido rápidamente en los últimos años en las organizaciones laborales, en especial a partir de la década de los 80 del siglo pasado. Entre los motivos que justifican su desarrollo se encuentra el hecho de que el término coaching se percibe menos problemático y amenazante para las organizaciones que otros términos de origen más psicológico asociados a problemas de salud mental. El término coach se asocia al deporte, al entrenamiento, o al asesoramiento, y no a la salud mental. Una organización que consulta a un psicólogo se puede percibir como una

"La realización de este estudio ha sido subvencionada por el Ministerio de Economía y Competitividad (MINECO), y fondos FEDER. Referencia proyecto: PSI2013-48185- $R^{\prime \prime}$ 
organización poco sana, con problemas, y carente de recursos. Una organización que consulta a un coach se percibe como una organización sana que busca potenciar sus recursos.

La práctica del coaching requiere tener ciertos conocimientos y competencias. Aunque dependiendo del propósito del coaching y del campo en que se desarrolle no es estrictamente necesario para su práctica profesional tener una formación de experto en Psicología, es recomendable que quienes lo desarrollen como actividad profesional tengan formación psicológica, pues existe una íntima relación entre psicología y coaching, ya que en las sesiones de coaching se tratará con cogniciones, emociones, actitudes, y conductas; todas ellas variables psicológicas. Por otra parte, determinadas aproximaciones al coaching sí hacen muy recomendable que el profesional que lo desarrolle sea un psicólogo.

En los siguientes puntos se desarrollarán algunos conceptos, reflexiones y estrategias que acercan el coaching a la psicología. El estudio establece algunas de las diferencias más relevantes entre coaching y terapia, para continuar presentando una de las aproximaciones más relevantes para la práctica del coaching desde una base psicológica: el coaching cognitivo-conductual (CCC). Esta aproximación resulta de especial utilidad aplicada debido a que permite redirigir los principios de la terapia cognitivo-conductual para potenciar los recursos de personas con bajos niveles de disfunción. El estudio concluye con algunas recomendaciones para aplicar el CCC en la prevención de los riesgos psicosociales en el trabajo y en la mejora de la calidad de vida laboral.

\section{Coaching y terapia}

El coaching se puede definir como el arte de facilitar el rendimiento, el aprendizaje, y el desarrollo de otra persona (Downey, 1999, p. 15). Es un proceso de acompañamiento en el que un experto (coach) aplica un conjunto de estrategias para que otra persona (coachee) mejore su situación personal y/o profesional. Esta mejora se consigue optimizando los recursos personales del coachee (habilidades, competencias, destrezas) durante el proceso de acompañamiento.

El objetivo de este proceso interpersonal es ejercer una influencia positiva por parte del experto para mejorar el comportamiento y el desempeño del cliente o coachee. Pero, el experto debe mantener una posición de neutralidad a lo largo de todo el proceso para no interferir en la toma de decisiones del cliente, pues su papel no es el de consultor, tutor o mentor. El experto no aconseja de modo directivo sobre cómo hacer las cosas.

El coaching se puede centrar en cualquier faceta de la vida de una persona para ayudarle en su crecimiento personal. Una de las cualidades del experto en coaching es su habilidad para ofrecer retroinformación continua al cliente con el fin de facilitar su aprendizaje y su desarrollo personal y profesional. Surge como una necesidad ante situaciones personales y laborales que hacen aconsejable la presencia de un profesional para acompañar el proceso de transformación y desarrollo de la persona.

Entre las funciones del coach están fomentar la adhesión a objetivos comunes, y la planificación sistemática del proceso de coaching. Además, debe enseñar al cliente a tener una visión de futuro, y le aporta he- 
rramientas y métodos para resolver problemas. El coach debe conseguir que el cliente combine planes realistas actuales con una visión de futuro también realista sobre la base de la esperanza y la energía para vivir plenamente, para resistir a la adversidad, y asumir compromisos consigo mismo y con los demás (Gil-Monte, 2014). Los temas sobre los que volverá el coach de forma recurrente serán: los valores, las relaciones, y la coherencia (Launer, 2011).

El coach debe realizar esas funciones sin recurrir a elementos de terapia, lo que en ocasiones resulta difícil de realizar debido a que la línea entre coaching y terapia puede ser borrosa y difícil de delimitar. Por ese motivo, el psicólogo se presenta como el profesional más cualificado para el ejercicio del coaching, en especial si se trata de ámbitos o temas relacionados con la salud y desde aproximaciones con base psicológica. Lo que no implica excluir otras aproximaciones al coaching y su desarrollo por otros profesionales.

Un criterio para diferenciar coaching y terapia es que mientras que la psicoterapia tiene que ver principalmente con tratamientos patológicos, el coaching trata con la mejora de las competencias y las experiencias vitales del individuo. Los clientes que buscan ayuda en sesiones de coaching suelen ser individuos con bajos niveles de patología y altos niveles de funcionalidad, mientras que los que asisten a terapia generalmente tienen problemas psicopatológicos y baja funcionalidad. El coaching puede tener efectos terapéuticos, pero no intenta cambiar estructuras psicológicas sino favorecer el desarrollo personal del cliente. El coach deberá identificar los recursos del cliente que facilitarán los comportamientos de éxito.
La psicoterapia buscar resolver un problema, y el cliente es considerado una persona que tiene una patología, una disfunción, o que busca resolver un problema de salud. El terapeuta es visto como un experto con conocimientos científicos en un determinado campo de la salud. El coaching tiene que ver con un aumento de las competencias, del desempeño, o de la experiencia de vida, más que tratar alguna disfunción o problema de salud. El terapeuta se centra en procesos internos, sentimientos, hechos pasados, procesos inconscientes, y desarrolla cambios de trastornos cognitivos para ayudar a los clientes a cambiar su comportamiento. El coaching consiste en acompañar al coachee en el proceso de superación de sus limitaciones personales. El coach suele recurrir al método socrático para que el coachee encuentre en sí mismo los elementos para mejorar como individuo y para alcanzar objetivos, al tiempo que maneja una situación externa. No se centra tanto en el individuo. Coach y coachee descubren conjuntamente cómo se puede avanzar mediante el análisis de la situación, la identificación de retos, de obstáculos y de facilidades, y diseñando un plan de acción (Gil-Monte, 2014).

\section{Bases teóricas del CCC}

Como se ha indicado más arriba el CCC se basa en los principios de las técnicas cognitivo-conductuales para potenciar los recursos de personas con bajos niveles de disfunción.

Las técnicas cognitivo-conductuales se caracterizan porque entrenan al individuo para que detecte y maneje sus pensamientos irracionales, pues parten del supuesto de que el origen de las disfunciones del comportamiento son una serie de ideas o 
pensamientos irracionales que ocasionan que los individuos tergiversen la realidad y que realicen procesos de atribución causal interna, ocasionando crisis de ansiedad. Entre los pensamientos irracionales más característicos y frecuentes se encuentran los siguientes (McKay, Davis y Fanning, 1985): (1) Filtraje o abstracción selectiva (2) pensamiento polarizado, (3) sobregeneralización, (4) interpretación del pensamiento, (5) visión catastrófica, (6) personalización, (7) falacia de control, (8) falacia de justicia, (9) razonamiento emocional, (10) falacia del cambio, (11) etiquetas globales, (12) culpabilidad, (13) los debería, (14) tener razón, y (15) falacia de la recompensa divina.

Una vez identificados esos pensamientos, las situaciones que los generan, y las emociones resultantes, el terapeuta procede a identificar los pensamientos adecuados o las creencias racionales que se dan en esas mismas situaciones, y que no tienen consecuencias negativas para el individuo. Entonces, modifica las creencias irracionales y entrena al individuo en estrategias que le permitan manejar del problema y la ansiedad que deriva de él.

Por tanto, el objetivo de las técnicas cognitivo-conductuales es ayudar al individuo a lograr un alivio de los síntomas del estrés reestructurando los pensamientos que hacen crónica la ansiedad, la depresión, la culpa, la angustia, la cólera, etc.

El desarrollo del CCC está vinculado a dos técnicas cognitivo-conductuales: el entrenamiento en resolución de problemas y a la terapia cognitivo-conductual (Kodish, 2002; Neenan, 2008):

(1) El entrenamiento en resolución de problemas (D'Zurilla, 1993; McKay, Davis y
Fanning, 1985) ayuda a las personas a resolver problemas y a mejorar su toma de decisiones. Para ello, facilita el reconocimiento del problema y su identificación, inhibiendo al mismo tiempo la tendencia de responder impulsivamente ante él. Facilita también el desarrollo de alternativas a la situación, ofrece estrategias para analizar las diferentes alternativas y ponderarlas, y propone un procedimiento para escoger la respuesta más adecuada al problema y verificar su adecuación.

(2) La terapia cognitivo-conductual (Gavino, 2002; Beck, 2000; Bas y Andrés, 1994) parte del supuesto de que para manejar la información del entorno los individuos lo simplifican mediante un procesamiento selectivo de la información y con la creación de esquemas cognitivos, del tipo de los estereotipos. Por tanto, la influencia del entorno sobre el comportamiento de las personas no se debe a las características objetivas de ese entorno, sino a la interpretación que las personas hacen de él. El estrés y la ansiedad se consideran resultado de un sesgo sistemático de la visión que la persona tiene del mundo.

El CCC (Palmer y Szymanska, 2007; Williams, Edgerton y Palmer, 2010) combina el uso de las técnicas de solución de problemas junto con actuaciones y estrategias cognitivo-conductuales en personas sin patología clínica para conseguir que alcancen objetivos reales. Se trata de una intervención limitada en el tiempo, orientada hacia el cumplimiento de metas, y enfocada en el presente. Esta aproximación busca la resolución de problemas para ayudar a los clientes en situaciones de bloqueo emocional, psicológico, o conductual, de manera que puedan seguir progresando en la consecución de objetivos. 


\section{Desarrollo del CCC}

Existen dos premisas básicas en el CCC: (1) Las personas pueden desarrollar destrezas para resolver problemas de manera eficaz en determinadas situaciones y, aunque les hayan funcionado, pueden ser incapaces de aplicarlas en otras situaciones. (2) Las emociones y cogniciones de un individuo en una situación vienen determinadas en mayor medida por sus creencias y por cómo percibe la situación, no por las características objetivas de la situación.

Además, el CCC considera que las emociones negativas que pueden surgir de la unión de ambas premisas, y que favorecen la aparición de ansiedad, pueden afectar también al comportamiento de la persona y a su capacidad para realizar las tareas. Teniendo presente esas circunstancias, el CCC pretende ayudar a las personas a que tomen conciencia de sus pensamientos limitadores, a mejorar sus destrezas para resolver problemas, y a modificar las creencias que limitan el rendimiento, que generan estrés, y que bloquean la consecución de objetivos. En el contexto laboral el acercamiento se puede centrar en mejorar el rendimiento en situaciones de tensión.

El término creencias irracionales no se suele utilizar en coaching debido a que los clientes perciben el término irracional como peyorativo. Se prefieren términos como pensamientos erróneos o pensamientos que interfieren el rendimiento.

Los objetivos del CCC son los siguientes:

(1) Ayudar al coachee a establecer objetivos realistas.

(2) Resolver problemas y dificultades.

(3) Ayudar a la coachee a adquirir destrezas y estrategias de afrontamiento constructivas.
(4) Ayudar al coachee a resolver los pensamientos y creencias irracionales (pensamientos erróneos) que le generan estrés, y que interfieren sobre su comportamiento.

(5) Ayudar al coachee a desarrollar destrezas para pensar en positivo, de manera que disminuyan sus niveles de estrés y mejore su rendimiento.

(6) Ayudar al coachee a ser su propio coach.

Para alcanzar esos objetivos se desarrollan las siguientes acciones:

1. Repasar brevemente con el coachee su estado actual: ¿Cómo te has encontrado recientemente?

2. Negociar un plan para la sesión: ¿Qué deseas que hagamos en la sesión de hoy?

3. Revisar las tareas realizadas entre sesiones: ¿Qué has hecho sobre las tareas fijadas en la sesión anterior hasta la de hoy?

4. Identificar el problema para la sesión actual: ¿Qué tenemos que hacer hoy?

5. Negociar las tareas entre dos sesiones.

6. Ofrecer retroinformación sobre la sesión del día: ¿Quieres que comentemos algo sobre la sesión de hoy?

Palabras como obligaciones o deberes deben ser evitadas en las sesiones de coaching debido a que pueden tener recuerdos negativos para algunos clientes. Revisar las tareas entre sesiones negociadas o asignadas en sesiones previas es un aspecto clave del coaching, pues al revisarlas se envía al cliente un mensaje indicándole que es importante realizarlas. También puede ser relevante recordarle al cliente lo impor- 
tante que son las horas fuera de sesión, en las que se desarrollan los planes de acción negociados y establecidos, pues los problemas que surgen al intentar desarrollar esos planes de acción también resultan útiles para el cliente.

\section{Preguntas guía y estrategias que puede utilizar el coach para analizar los pensamientos distorsionados y sus consecuencias.}

Durante el proceso de coaching, el coach y el coachee trabajan de manera conjunta y en colaboración para enfocar los problemas de manera diferente y cambiar su perspectiva. El procedimiento consiste en utilizar preguntas basadas en un proceso de autorreflexión sistemática y en el razonamiento inductivo (de lo particular a lo general) siguiendo el método socrático. Este proceso se puede utilizar para ayudar a identificar los pensamientos distorsionados y modificarlos. Algunas de las preguntas socráticas que puede utilizar el coach para analizar la validez de esos pensamientos son las siguientes:

(1) ¿Dónde está la evidencia de tus creencias?

(2) ¿Cómo te está afectando este tipo de pensamiento? ¿Cómo influye sobre ti?

(3) ¿No estarás yendo directamente a las conclusiones?

(4) ¿Tienes alguna evidencia para cuestionar tus creencias?

(5) ¿No crees que te estás centrando en tus debilidades y negando tus fortalezas?

(6) ¿Cuáles son las ventajas e inconvenientes de pensar de esta manera?

(7) ¿No estarás tomando las cosas de una manera demasiado personalizada?
(8) ¿No te parece que estás pensando en términos de todo o nada?

(9) ¿No te parece que estás aplicando un doble criterio?

(10) ¿No estarás sobrevalorando las posibilidades de que ocurra algo malo?

(11) ¿Estarás prediciendo el resultado en lugar de verlo cuando ocurre?

(12) ¿Estás esperando la perfección absoluta?

(13) Qué estrategias prácticas puedes adoptar para convencerte de lo inadecuado de tus pensamientos?

En el siguiente punto se presentan los modelos más relevantes de CCC con el fin de ofrecer al psicólogo un referente y un encuadre que facilite el ejercicio de su actividad profesional como coach. Se revisan tres modelos: PRACTICE, SPACE, y ABCDEF.

\section{Algunos modelos de CCC}

\section{El modelo PRACTICE.}

Tomando como referencia la técnica de solución de problemas desarrollada por D'Zurilla y colaboradores (D'Zurilla y Goldfried, 1971; D’Zurilla y Nezu, 1982), en la década de los 80, B. Wasik desarrolló un modelo de solución de problemas estructurado en siete pasos que se ha aplicado en counseling, psicoterapia, gestión, entrenamiento y coaching. Los pasos desarrollados por Wasik son: (1) Identificación del problema, (2) selección de objetivos, (3) generación de alternativas, (4) análisis de consecuencias, (5) toma de decisiones, (6) ejecución de las soluciones, y (7) evaluación.

Posteriormente, Palmer (2007; 2008) de- 
sarrolló el modelo PRACTICE, que es una adaptación del modelo de Wasik. Siguiendo este modelo, y antes de abordar el primer paso, en la primera sesión de coaching se le da al cliente la opción de hablar sobre él sin necesidad de abordar sus problemas o sus preocupaciones, de manera que el coach consigue aprender más sobre el cliente y sobre sus circunstancias y su contexto social. El coach debe llamar la atención del clien-

Tabla 1. Posibles preguntas para progresar en las sesiones de coaching siguiendo el modelo PRACTICE.

\begin{tabular}{ll}
\hline Pasos & Preguntas y acciones \\
\hline 1. Identificación del problema & - ¿Cuál es el problema o el tema que deseas discutir o tratar? \\
(Problem identification) & - ¿Qué te gustaría cambiar? \\
& - ¿Hay algún momento en que no lo veas como un problema? \\
& - ¿Cómo podríamos saber si la situación ha mejorado? \\
& - En una escala de 0 a 10, donde 0 es "Nada" y 10 es "Se ha resuelto" ¿Cómo de \\
& cerca estás a día de hoy para resolver ese problema o asunto? \\
& - ¿Consideras que se puedes estar viendo la situación distorsionada o la podrías \\
& ver de forma diferente? \\
& - ¿Puedes imaginar despertarte mañana y que este problema o preocupación ya \\
& no exista? ¿Qué sería diferente para ti y por qué?
\end{tabular}

2. Desarrollo realista de objeti- ¿Qué deseas conseguir? Vamos a identificar y desarrollar objetivos específicos. vos relevantes

(Realistic, relevant goals deve-

loped)
3. Generación de soluciones ¿Cuáles son tus opciones? Escríbelas alternativas
(Alternative solutions genera- ted)
4. Consideración de consecuen- - ¿Qué podría pasar? cias - ¿Cuán útil es cada solución posible?
(Consideration of consequen- Apliquemos una escala de utilidad para cada solución, en la que 0 indica que la ces) Solución "No es nada útil" y 10 significa que la solución "Es extremadamente útil"

5. Objetivo/-s más fácil de al- Ahora que hemos considerado todas las posibles soluciones, ¿cuál es la solucanzar ción más práctica?

(Target most feasible solution)

6. Aplicación de la/-s solución/- $\quad$ Poner en práctica la solución elegida siguiendo un proceso de pasos sucesivos es elegida/-s. que sean manejables.

(Implementation of Chosen solution)

7. Evaluación

(Evaluation)
- ¿Cuál fue el nivel de éxito? Valoración de 0 a 10.

- ¿Qué podemos aprender?

- ¿Podemos terminar el coaching ahora o deseas tratar, abordar o discutir otro tema u otro problema? 
te sobre cualquier información que ponga de manifiesto sus competencias, esfuerzos y cualidades, insistiendo en ejemplos de esas competencias en situaciones pasadas. También puede recurrir a presentar las situaciones negativas como excepciones que confirman las fortalezas del cliente cuando se trate de problemas menores. Durante las reuniones, y a lo largo de todo el proceso de coaching, se deben de realizar preguntas para que el cliente tome conciencia del punto en el que se encuentra, cómo está progresando, y lo que debería hacer para mejorar. Un ejemplo de preguntas (Palmer, 2008) se muestran en la Tabla 1.

Una vez que se tiene cierto grado de experiencia en la aplicación de este modelo de siete pasos se puede optar por seguir un modelo más breve con el fin de acelerar el proceso de búsqueda de soluciones. Es el caso de los modelos:

- PIE:

Problem definition (Definición del problema)

Implement a solution (Puesta en práctica de la solución)

Evaluate outcome (Evaluación de resultados)

- STIR:

Select problem (Selección del problema)

Target a solution (Identificación de la solución)

Implement a solution (Puesta en práctica de la solución)

Review outcome (Revisión de resultados)

\section{EI modelo SPACE.}

El modelo SPACE se desarrolló para aplicarlo en coaching, terapia, y manejo del estrés (Edgerton y Palmer, 2005). Permite representar las diferentes facetas del individuo implicadas en los procesos psicológicos. Este modelo considera los aspectos sociales y fisiológicos del coachee junto con sus conductas, cogniciones y emociones, en el proceso de cambio para que el coachee alcance sus objetivos. El esquema del modelo es el siguiente:

Social context (contexto social)

Physiology (fisiología)

Action (comportamientos)

Cognition (cogniciones)

Emotion (emociones)

Por ejemplo, un individuo que va a realizar una presentación en público (contexto social) puede percibir que la situación es difícil (cognición). Esta percepción negativa probablemente le creará ansiedad (emoción), con la aparición de sudoración e incremento de la tensión arterial (fisiología), lo que le puede llevar marcharse para evitar la situación (acción/conducta). El modelo SPACE crea diagramas de este ciclo para facilitar su comprensión y manejo al coachee (Figura 1).

Mediante el modelo SPACE también se pueden representar aspectos positivos del individuo, lo que permite representar los resultados de la intervención (Figura 2).

\section{El modelo ABCDEF.}

Albert Ellis desarrolló en la segunda mitad del siglo pasado el modelo ABC sobre las emociones (Ellis, 1958) (en castellano se puede consultar, Ellis, 1980). En este modelo, la A representa la situación o 


\section{Contexto social o situación}
(afrontar un problema de salud)

\section{Sudoración, palpitaciones, temblor de manos, hormigueo en estómago, etc.}

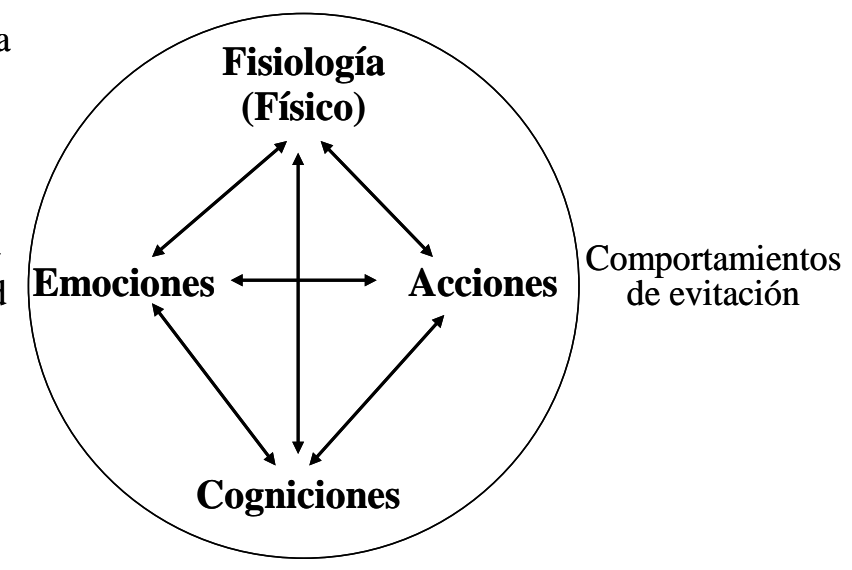

No voy a ser capaz

Todo me pasa a mí

Es imposible

Va a salir todo mal el evento negativo que activa al individuo (Activating event), la B representa las creencias que el individuo desarrolla sobre ese acontecimiento (Beliefs), y la C las consecuencias emocionales, fisiológicas y conductuales que el evento y su interpretación tiene sobre la persona (Consequences).

Ellis considera que el problema fundamental que subyace a los problemas es la elaboración de creencias rígidas, inflexibles, sin base empírica, poco lógicas y nada funcionales. A estas creencias las denomina creencias irracionales y las distingue de las inferencias que la gente hace considerando los hechos en otras situaciones.

Por ejemplo, una entrevista de trabajo en términos $\mathrm{ABC}$ sería:

\section{A (Situación activadora)}

- Entrevista de trabajo.

B (Creencias sobre A)

- Esta entrevista de trabajo va a ser difícil y desagradable.

- Tengo que hacerlo bien en la entrevista de trabajo.

- No podría soportar fallar.

C (Consecuencias)

- Ansiedad elevada.

- Pasearse por la sala de espera, hacerlo mal en la entrevista.

- Palpitaciones, sudoración, etc.

El modelo $\mathrm{ABC}$ incluye dos etapas adicionales: 
Figura 2. Representación del estado positivo del individuo mediante el modelo SPACE.

\section{Contexto social o situación}

Alto rendimiento

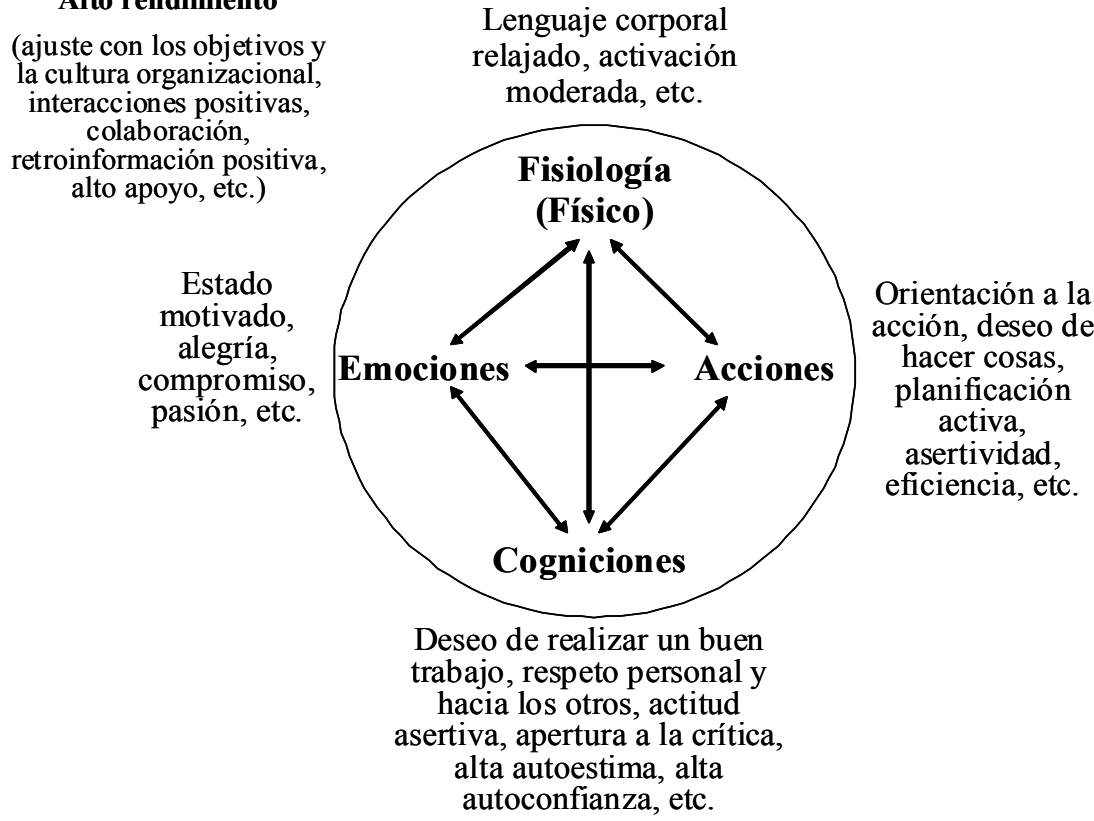

$\boldsymbol{D}$ (Debate de las creencias irracionales)

$\boldsymbol{E}$ (nuevo acercamiento Efectivo para tratar con la situación o los eventos activadores)

En la práctica, durante la sesión de coaching, el coach deberá trabajar junto con el cliente anotando sus pensamientos $\mathrm{y}$ creencias irracionales para ayudarle a entender el modelo cognitivo y los procesos que subyacen.

Con frecuencia, al inicio del proceso de coaching, el coachee ya piensa que son sus pensamientos los que contribuyen en mayor medida al bajo rendimiento y no la situación. Sin embargo, la comprensión de los aspectos emocionales necesita más tiempo para desarrollarse debido a que el coachee debe poner en práctica los nuevos recursos de ayuda para generar pensamientos y creencias útiles, y observar como disminuye el nivel de estrés y mejora el rendimiento.

Palmer (2002) añadió una sexta etapa a este modelo para aplicarlo en procesos de coaching. Esta etapa la denomina $\mathbf{F}$, y representa Focalizarse en los objetivos personales y laborales futuros y en el aprendizaje del proceso $\mathrm{ABCDE}$ que puede mejorar el rendimiento y proteger del estrés.

Siguiendo con el ejemplo de la entrevista de trabajo: 


\section{D (Debate)}

- ¿La entrevista de trabajo es una amenaza real? Siendo realistas, ¿será tan difícil y desagradable?

- ¿Por qué tienes que hacerlo bien? Es preferible hacerlo bien, pero si no tampoco es el final.

- ¿Es cierto que no puedes fallar? Has superado otras situaciones a pesar de que no todas eran agradables.

\section{$\boldsymbol{E}$ (Acercamiento efectivo)}

- El cliente centra las creencias en los objetivos y en la entrevista, vive el interés y no la ansiedad, para de pasear por la sala, reduce las respuestas fisiológicas.

\section{F (Focalizar el futuro)}

- El foco permanece en los logros conseguidos. El cliente aprende a ser menos ansioso atendiendo al proceso de entrevista. Aprende a no ser tan perfeccionista y a no exigirse hacerlo todo perfecto.

El modelo completo incluye el desarrollo inicial de objetivos específicos, lo que lleva a un modelo G-ABCDEF. En coaching este modelo se conoce como el modelo de estrés, rendimiento, resiliencia y bienestar. De esta manera, se va más allá del modelo original, y se pasa de tratar sólo con los problemas clínicos del lugar de trabajo a centrarse en los aspectos positivos, tales como la mejora del rendimiento.

\section{Manejo del estrés y la ansiedad desde el coaching}

En coaching es necesario distinguir entre individuos que presentan estrés positivo (eustrés) y quienes presentan estrés negativo (distrés), y puede surgir la pregunta ¿cómo puedo saber si el cliente presenta un problema de salud o una patología que deba ser tratada mediante terapia o, por el contrario, optar por un proceso de coaching? ¿qué hacer en esos casos?

Como se ha indicado anteriormente, es importante tener presente que el coaching se desarrolla con personas sanas, sin patología mental, y con bajo niveles de disfunción. Ante la sospecha de que exista un problema de salud relacionado con el estrés, el coach con una titulación en psicología deberá optar por un abordaje terapéutico, y si carece de esa formación deberá remitir al cliente a un profesional especializado que aborde esos problemas. Nunca deberá resolverlos mediante el proceso de coaching.

El estrés crónico y la ansiedad que lo acompañan pueden derivar en problemas de salud importantes como la depresión y, por tanto, salirse del campo de actuación del coaching. Una persona que presenta un problema de salud no está preparada en ese momento para participar en un proceso de coaching, entre otras cosas debido a que puede ser incapaz de marcarse objetivos y cursos de acción. Ante una situación de este tipo es muy importante comunicarle al cliente lo antes posible nuestro punto de vista, y recomendarle que aborde el tratamiento del problema clínico como paso previo a iniciar o a continuar con el proceso de coaching. El coaching no es una terapia $y$ el coach no es un terapeuta.

Saiz y Laínsa (2010) presentan algunos síntomas de alerta que un coach debe tener en cuenta para decidir si se inicia o se continúa el proceso de coaching o, por el contrario, se remite al coachee a terapia. Señalan estos autores que esas alerta no son válidas en sí mismas y de forma aislada, pues hay que tener en cuenta la presencia simultánea 
de tres o cuatro, y durante un periodo consecutivo de más de tres meses. Las alertas a tener en cuenta son las siguientes (ver también Bayón, Cubeiro, Romo y Sáinz, 2006): (1) El cliente parece depresivo. (2) Es excesivamente dependiente de otros. (3) $\mathrm{Su}$ vida es extremadamente caótica. (4) Se observa una pauta de relaciones muy inestable con los demás. (5) Sus creencias no son consecuentes con la realidad. (6) No se fía en absoluto de los demás. (7) Está siempre enfadado o agresivo. (8) Piensa en comportamientos autodestructivos. (9) Es extremadamente egocéntrico. (10) Dice cosas como: "eres el/la único/a que te preocupas por mí”. (11) A estos síntomas de alerta se añaden otros de corte psicosomático como la presencia de: palpitaciones, taquicardia, tensión arterial alta, golpes de calor, dolor de cabeza frecuente, ahogos, respiración rápida y superficial, opresión en el tórax, nauseas, vómitos, diarrea, aerofagia y molestias digestivas, micción frecuente, enuresis, eyaculación precoz, frigidez, impotencia, elevada tensión muscular, temblores, hormigueos, dolor de cabeza, sequedad de boca, sudoración excesiva, mareo, preocupación excesiva, sensación de estar desbordado, inquietud o impaciencia, tendencia a la fatiga con facilidad, dificultad para concentrarse, irritabilidad, intolerancia, agresividad, alteraciones de sueño, bajas laborales frecuentes, y sentimientos de culpa por sus actos y comportamientos o pensamientos.

\section{¿Qué se debe trabajar desde el coaching con relación a los riesgos psicosociales en el trabajo, el estrés laboral y la ansiedad?}

Los factores laborales que contribuyen a desarrollar los niveles de estrés son numerosos, y con frecuencia el individuo no es consciente de su presencia, ni de que puede estar utilizando estrategias de afrontamiento disfuncionales. En el proceso de CCC se debe trabajar sobre la identificación de las fuentes de estrés, o riesgos psicosociales, y sobre la forma en que el cliente afronta esas situaciones, incluyendo el manejo de las emociones, para aprender a autogestionar el estrés y mejorar la calidad de vida.

Para que el coachee desarrolle un plan de acción el coach deberá clarificarle los objetivos, orientarlo, y estructurar su aprendizaje. También puede contribuir a la clarificación de la situación organizacional, y potenciar los recursos de comprensión del problema. En los estudios de Palmer (2002) y de Wright (2007) se pueden consultar ejemplos de intervención organizacional mediante el coaching para prevenir el estrés laboral.

Como conclusión, hay que señalar que el coahing ayuda a tomar decisiones en momentos difíciles, y a enfrentarse de un modo saludable a los cambios. Es útil para la detección y resolución de problemas personales o laborales que pueden afectar el rendimiento y satisfacción del empleado, ayuda al trabajador a adoptar una actitud constructiva en situaciones conflictivas, aumenta su autoconfianza, su autoestima y le confiere un mayor equilibrio emocional. El coaching puede ser incorporado a los programas de ayuda al empleado en la prevención de riesgos psicosociales dentro del nivel de intervención individual (ver, Solé y Balduque, 2007), pues esta técnica se caracteriza por ser un procedimiento que facilita a los empleados disponer de recursos personales para afrontar con más seguridad y con menor coste emocional las situaciones de riesgo psicosocial. 


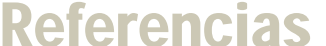

Bas. F., y Andrés, V. (1994). Terapia cognitivo conductual de la depresión: un manual de tratamiento. Madrid: Fundación Universidad-Empresa.

Bayón, F., Cubeiro, J.C., Romo, M., y Sáinz, J.A. (2006). Coaching realmente. Madrid: Pearson.

Beck, J.S. (2000). Terapia cognitiva: conceptos básicos y profundización. Barcelona: Gedisa.

D'Zurilla, T.J. (1993). Terapia de resolución de conflictos: competencia social, un nuevo enfoque en la intervención clínica. Bilbao: Desclée de Brouwer.

D'Zurilla, T.J., y Goldfried, M.R. (1971). Problem solving and behavior modification. Journal of Abnormal Psychology, 78, 107-126.

D'Zurilla, T.J., y Nezu, A.M. (1982). Social problem solving in adults. En P.C. Kendall (Ed.), Advances in cognitive-behavioral research and therapy (Vol. 1, pp. 201-274). New York: Academic Press.

Downey, M. (1999). Effective coaching. London: Orion Business Books.

Edgerton, N., y Palmer, S. (2005). SPACE: A psychological model for use within cognitive behavioural coaching, therapy and stress management. The Coaching Psychologist, 1, 25-31.

Ellis, A. (1980). Razón y emoción en psicoterapia. Bilbao: Desclée de Brouwer.

Ellis, A. (1958). Rational psychotherapy. Journal of General Psychology, 59, 35-49.

Gavino, A. (2002). Guía de ayuda al terapeuta cognitivoconductual. Madrid: Pirámide.

Gil-Monte, P.R. (2014). La intervención en riesgos psicosociales: coaching y apoyo social en el trabajo. En P.R. Gil-Monte (Coord.), Manual de Psicosociología aplicada al trabajo y a la prevención de los riesgos laborales (pp., 515-542). Madrid: Pirámide.

Kodish, S. P. (2002). Rational emotive behavior coaching. Journal of Rational-Emotive \& Cognitive-Behavior Therapy, 20, 235-246.

Launer, V. (2011). Coaching. Un camino hacia nuestros éxitos (4 ${ }^{\mathrm{a}}$ ed.). Pirámide: Madrid.

McKay, M., Davis, M., y Fanning, P. (1985). Técnicas cognitivas para el tratamiento del estrés. Barcelona: Martínez Roca.

Neenan, M. (2008). From cognitive behaviour therapy (CBT) to cognitive behaviour coaching (CBC). Journal of Rational-Emotive \& Cognitive-Behavior Therapy, 26, 3-15.
Palmer, S. (2002). Cognitive and organisational models of stress that are suitable for use within workplace stress management/prevention coaching, training and counselling settings. Rational Emotive Behaviour Therapist, 10, 15-21.

Palmer, S. (2007). PRACTICE: A model suitable for coaching, counselling, psychotherapy and stress management. The Coaching Psychologist, 3, 71-77.

Palmer, S. (2008). The PRACTICE model of coaching: towards a solution-focused approach. Coaching Psychology International, 1, 4-8.

Palmer, S., y Szymanska, K. (2007). Cognitive behavioural coaching. En S. Palmer y A. Whybrow (Eds.), Handbook of coaching psychology (pp. 86-117). New York, NY: Routledge.

Saiz, R., y Laínsa, N. (2010). Coaching para el estrés. En F. Bayón (Coord.), Coaching hoy. Teoría general del coaching (pp. 366-376). Madrid: Ramón Areces.

Solé, M.D., y Balduque, M. (2007). El programa de ayuda al empleado (EAP): intervención individual en la prevención de riesgos psicosociales. Nota Técnica de Prevención 780 del INSHT. Madrid: Instituto Nacional de Seguridad e Higiene en el Trabajo. Disponible en (12 marzo 2014): http://www.insht.es/InshtWeb/Contenidos/Documentacion/FichasTecnicas/NTP/Ficheros/751a785/780\%20.pdf

Williams, H., Edgerton, N., y Palmer, S. (2010). Cognitive behavioural coaching. En E. Cox, T. Bachkirova y D. Clutterbuck (Eds.), The complete handbook of coaching (pp. 37-53). London: Sage.

Wright, J. (2007). Stress in the workplace: A coaching approach. Journal of Prevention, Assessment \& Rehabilitation, 28, 279-284.
Fecha de recepción: 7/01/2014 Fecha de aceptación: 2/04/2014 